\title{
Application of He's Homotopy Perturbation Method to Fractional Diffusion Equations
}

\author{
Subir Das, Praveen Kumar Gupta, Vinod Sankar Pandey, and Kabindra Nath Rai \\ Department of Applied Mathematics Institute of Technology, Banaras Hindu University, \\ Varanasi - 221005 , India
}

Reprint requests to S. D.; E-mail: subir_das08@hotmail.com

Z. Naturforsch. 65a, 53 - 58 (2010); received January 12, 2009 / revised May 9, 2009

\begin{abstract}
In this paper, the approximate analytical solutions of a general diffusion equation with fractional time derivative in the presence of a linear external force are obtained with the help of the homotopy perturbation method (HPM). The explicit solutions of the problem for the initial condition as a function of $x$ have been obtained. It reveals that a few iterations are needed to obtain accurate approximate analytical solutions. The numerical calculations are carried out when the initial conditions are like exponential and periodic functions and the results are depicted through graphs. The examples prove that the method is extremely effective due to its simplistic approach and performance.
\end{abstract}

Key words: Fractional Diffusion Equation; Homotopy Perturbation Method; Inital Value Problem; Mittag-Leffler Function.

\section{Introduction}

The simple fractional diffusion equation is given by

$$
\frac{\partial^{\alpha} u(x, t)}{\partial t^{\alpha}}=c^{2} \frac{\partial^{2} u(x, t)}{\partial x^{2}}, \quad 0<\alpha<1 .
$$

For the whole hierarchies of moments, $M_{k}(t)=\left\langle x^{k}(t)\right\rangle$ have the same time dependence as in the case of fractional Brownian motion (FBM), which can be observed from the expressions

$$
M_{2 k}(t)=\frac{\Gamma(2 k+1)}{\Gamma(3 k+1)} t^{\alpha k}, M_{2 k+1}(t)=M_{2 k+1}^{\mathrm{FBM}}(t)=0 .
$$

Though they have similar scaling properties, it is seen that $u(r, t) \neq u_{\mathrm{FBM}}(r, t)$ and they have different asymptotic decays. This idea is also true for a general fractional diffusion equation.

The analytical fractional diffusion equation is governed by the equation

$$
\begin{aligned}
& \frac{\partial^{\alpha} u(x, t)}{\partial t^{\alpha}}=\frac{\partial^{2} u(x, t)}{\partial x^{2}}-\frac{\partial}{\partial x}(f(x) u(x, t)), \\
& 0<\alpha \leq 1
\end{aligned}
$$

with the initial condition

$$
u(x, 0)=g(x)
$$

where $\frac{\partial^{\alpha}}{\partial t^{\alpha}}(\bullet)$ is the Caputo derivative of order $\alpha$, $u(x, t)$ represents the probability density function of finding a particle at $x$ in the time $t, f(x)$ is the external force. This type of problem has already been solved by Saha Ray and Bera [1] for some particular cases with initial conditions $u(x, 0)=1$ and $X$, when $\alpha=\frac{1}{2}$ and $f(x)=-x$ by using the Adomian decomposition method. But the disadvantage of the Adomian method is that the solution procedures of finding Adomian polynomials are cumbersome. Recently, Das [2] has solved the problem of general order $\alpha$ with $u(x, 0)=1, x, x^{2}$, and $f(x)=-x$, by using the powerful variational iteration method (VIM). In this solution the authors have claimed that the displacement decreases with the increase of Brownian motions $\alpha=\frac{1}{3}, \frac{1}{2}, \frac{2}{3}$ and the displacement increases with the increase of the degree of the polynomials of $u(x, 0)$. But to the best of the authors' knowledge, the solution of the general equation (3) with the initial condition as any function of $x$ given in (4) has not yet been solved by any researcher. No analytical method for solving such equations was available before 1998; this is also true for linear fractional differential equations. In 1998, the variational iteration method was first proposed to solve fractional differential equations with greatest success [3]. Following this work, many authors such as Odibat and Momani [4], Momani and Odibat [5], Ganji et al. [6], Das [7] etc. found VIM is an effective way to solve fractional equations: both 
linear and nonlinear. In 2008, Momani et al. [8], Odibat and Momani [9] compared the solution procedure of VIM with HPM. In the present study the authors have solved (3) with the help of the homotopy perturbation method, which was first proposed by He [10,11] and subsequently implemented by other researchers [1225]. The approximate analytical solution of $u(x, t)$ is deduced for a number of particular cases. The main advantage of the HPM is that it reduces both nonlinear differential equations and fractional linear differential equations to a series of ordinary differential equations, which are easy to solve for any order of approximations, as and when required. Here a sincere attempt is made to solve the problem for the initial conditions $u(x, 0)=\cos x$ and $\mathrm{e}^{x}$, which is discussed in the Section 4 (Numerical Results and Discussion). So far as the authors' knowledge goes, this method is a novel technique to solve problems specified above.

\section{Solution of the Problem}

Equation (3) can be written in operator form as

$$
D_{t}^{\alpha} u=D_{x x} u-D_{x}(f(x) u), \text { where } D_{t}^{\alpha} \equiv \frac{\partial^{\alpha}}{\partial t^{\alpha}} .
$$

According to the homotopy perturbation method, we construct the following homotopy:

$$
D_{t}{ }^{\alpha} u=p\left[D_{x x} u-D_{x}(f(x) u)\right],
$$

where the homotopy parameter $p$ is considered as a small parameter $(p \in[0,1])$. In case $p=0$, (6) becomes a linear equation, $D_{t}^{\alpha} u=0$, which is easy to be solved [23-25]. Now applying the classical perturbation technique, we can assume that the solution of (3) can be expressed as a power series in $p$ as given below:

$$
u=u_{0}+p u_{1}+p^{2} u_{2}+p^{3} u_{3}+p^{4} u_{4}+\ldots .
$$

When $p=1$, (6) corresponds to (5) and (7) becomes the approximate solution of (5) i. e., of (3). The convergence of the method has been proved in [11]. Substituting (7) for (6), and equating the terms with the identical powers of $p$, we can obtain a series of equations:

$$
\begin{aligned}
& p^{0}: D_{t}{ }^{\alpha} u_{0}=0, \\
& p^{1}: D_{t}{ }^{\alpha} u_{1}=D_{x x} u_{0}-D_{x}\left(f(x) u_{0}\right), \\
& p^{2}: D_{t}{ }^{\alpha} u_{2}=D_{x x} u_{1}-D_{x}\left(f(x) u_{1}\right),
\end{aligned}
$$

$$
\begin{aligned}
& p^{3}: D_{t}{ }^{\alpha} u_{3}=D_{x x} u_{2}-D_{x}\left(f(x) u_{2}\right), \\
& p^{4}: D_{t}{ }^{\alpha} u_{4}=D_{x x} u_{3}-D_{x}\left(f(x) u_{3}\right),
\end{aligned}
$$

and so on.

The method is based on applying the operator $J^{\alpha}$ (the inverse of operator $D_{t}{ }^{\alpha}$ ) on both sides of (8) - (12). We obtain:

$$
\begin{aligned}
& u_{0}(x, t)=g(x) \\
& u_{1}(x, t)=\left[g^{(2)}(x)-f(x) g^{(1)}(x)-f^{(1)}(x) g(x)\right] \\
& \quad \frac{t^{\alpha}}{\Gamma(\alpha+1)}, \\
& u_{2}(x, t)=\left[g^{(4)}(x)-2 f(x) g^{(3)}(x)-4 f^{(1)}(x) g^{(2)}(x)\right. \\
& +\{f(x)\}^{2} g^{(2)}(x)-3 f^{(2)}(x) g^{(1)}(x) \\
& +3 f(x) f^{(1)}(x) g^{(1)}(x)-f^{(3)}(x) g(x) \\
& +
\end{aligned}
$$




$$
\begin{aligned}
& +58\left\{f^{(1)}(x)\right\}^{2} g^{(4)}(x)-36\{f(x)\}^{2} f^{(1)}(x) g^{(4)}(x) \\
& +\{f(x)\}^{4} g^{(4)}(x)-40 f^{(4)}(x) g^{(3)}(x) \\
& +80 f(x) f^{(3)}(x) g^{(3)}(x)+118 f^{(1)}(x) f^{(2)}(x) g^{(3)}(x) \\
& -50\{f(x)\}^{2} f^{(2)}(x) g^{(3)}(x)-80 f(x)\left\{f^{(1)}(x)\right\}^{2} g^{(3)}(x) \\
& +10\{f(x)\}^{3} f^{(1)}(x) g^{(3)}(x)+52 f(x) f^{(4)}(x) g^{(2)}(x) \\
& +86 f^{(1)}(x) f^{(3)}(x) g^{(2)}(x)-40\{f(x)\}^{2} f^{(3)}(x) g^{(2)}(x) \\
& -160 f(x) f^{(1)}(x) f^{(2)}(x) g^{(2)}(x)-40\left\{f^{(1)}(x)\right\}^{3} g^{(2)}(x) \\
& +39\left\{f^{(2)}(x)\right\}^{2} g^{(2)}(x)-6 f^{(5)}(x) g^{(2)}(x) \\
& +10\{f(x)\}^{3} f^{(2)}(x) g^{(2)}(x) \\
& +25\{f(x)\}^{2}\left\{f^{(1)}(x)\right\}^{2} g^{(2)}(x)-7 f^{(6)}(x) g^{(1)}(x) \\
& +19 f(x) f^{(5)}(x) g^{(1)}(x)+55 f^{(1)}(x) f^{(4)}(x) g^{(1)}(x) \\
& +85 f^{(2)}(x) f^{(3)}(x) g^{(1)}(x)-17\{f(x)\}^{2} f^{(4)}(x) g^{(1)}(x) \\
& -83 f(x) f^{(1)}(x) f^{(3)}(x) g^{(1)}(x) \\
& -51 f(x)\left\{f^{(2)}(x)\right\}^{2} g^{(1)}(x) \\
& -75\left\{f^{(1)}(x)\right\}^{2} f^{(2)}(x) g^{(1)}(x)-f^{(7)}(x) g^{(1)}(x) \\
& +3\{f(x)\}^{3} f^{(3)}(x) g^{(1)}(x) \\
& +30\{f(x)\}^{2} f^{(1)}(x) f^{(2)}(x) g^{(1)}(x) \\
& +15 f(x)\left\{f^{(1)}(x)\right\}^{3} g^{(1)}(x) \\
& +3 f(x) f^{(6)}(x) g(x)+10 f^{(1)}(x) f^{(5)}(x) g(x) \\
& +18 f^{(2)}(x) f^{(4)}(x) g(x)+11\left\{f^{(1)}(x)\right\}^{2} g(x) \\
& -3\{f(x)\}^{2} f^{(5)}(x) g(x)-17 f(x) f^{(1)}(x) f^{(4)}(x) g(x) \\
& -25 f(x) f^{(2)}(x) f^{(3)}(x) g(x)-3 f^{(1)}(x)\left\{f^{(2)}(x)\right\}^{2} g(x) \\
& -18\left\{f^{(1)}(x)\right\}^{2} f^{(3)}(x) g(x)-18 f^{(1)}(x)\left\{f^{(2)}(x)\right\}^{2} g(x) \\
& -\{f(x)\}^{3} f^{(4)}(x) g(x)+\{f(x)\}^{2} f^{(1)}(x) f^{(3)}(x) g(x) \\
& +4\left\{f^{(2)}(x)\right\}^{2} g(x)+11 f(x)\left\{f^{(1)}(x)\right\}^{2} f^{(2)}(x) g(x) \\
& \left.+\left\{f^{(1)}(x)\right\}^{4} g(x)\right] \frac{t^{4 \alpha}}{\Gamma(4 \alpha+1)},
\end{aligned}
$$$$
\text { where } f^{(r)}(x)=\frac{\partial^{r}}{\partial x^{r}}(f(x)) \text { and } g^{(r)}(x)=\frac{\partial^{r}}{\partial x^{r}}(g(x)) \text {. }
$$

Proceeding in this manner the components $u_{n}, n \geq 0$, of the HPM can be completely obtained and the series solutions are thus entirely determined.

Finally, we approximate the analytical solution $u(x, t)$ by the truncated series

$$
u(x, t)=\lim _{N \rightarrow \infty} \Phi_{N}(x, t),
$$

where $\Phi_{N}(x, t)=\sum_{n=0}^{N-1} u_{n}(x, t)$.

\section{Particular Cases}

Case I: If $f(x)=-x$ and $g(x)=1$, then

$$
\begin{aligned}
u(x, t)= & 1+\frac{t^{\alpha}}{\Gamma(\alpha+1)}+\frac{t^{2 \alpha}}{\Gamma(2 \alpha+1)} \\
& +\frac{t^{3 \alpha}}{\Gamma(3 \alpha+1)}+\frac{t^{4 \alpha}}{\Gamma(4 \alpha+1)}+\ldots \\
= & \sum_{r=0}^{\infty} \frac{t^{r \alpha}}{\Gamma(r \alpha+1)}=E_{\alpha}\left(t^{\alpha}\right),
\end{aligned}
$$

where $E_{\alpha}\left(t^{\alpha}\right)$ is the Mittag-Leffler function in one parameter. This result is the same as the result of Saha Ray and Bera [1] and Das [2].

Case II: If $f(x)=-x$ and $g(x)=x$, then

$$
\begin{aligned}
u(x, t)= & x+\frac{2 x t^{\alpha}}{\Gamma(\alpha+1)}+\frac{4 x t^{2 \alpha}}{\Gamma(2 \alpha+1)} \\
& +\frac{8 x t^{3 \alpha}}{\Gamma(3 \alpha+1)}+\frac{16 x t^{4 \alpha}}{\Gamma(4 \alpha+1)}+\ldots \\
= & \sum_{r=0}^{\infty} \frac{2^{r} t^{r \alpha}}{\Gamma(r \alpha+1)}=x E_{\alpha}\left(2 t^{\alpha}\right) .
\end{aligned}
$$

This result is the same as the results of Saha Ray and Bera [1] and Das [2].

Case III: If $f(x)=-x$ and $g(x)=x^{2}$, then

$$
\begin{aligned}
u(x, t)= & x^{2}+\frac{\left(2+3 x^{2}\right) t^{\alpha}}{\Gamma(\alpha+1)}+\frac{\left(8+9 x^{2}\right) t^{2 \alpha}}{\Gamma(2 \alpha+1)} \\
& +\frac{\left(26+27 x^{2}\right) t^{3 \alpha}}{\Gamma(3 \alpha+1)}+\frac{\left(80+81 x^{2}\right) t^{4 \alpha}}{\Gamma(4 \alpha+1)}+\ldots \\
= & \sum_{r=0}^{\infty} \frac{k_{1}{ }^{r} t^{r \alpha}}{\Gamma(r \alpha+1)}=E_{\alpha}\left(k_{1} t^{\alpha}\right),
\end{aligned}
$$

where $k_{1}{ }^{r}=x^{2}+\left(1+x^{2}\right)\left(3^{r}-1\right)$. This result is the same as the result of Das [2]. 
(a) $\mathrm{u}(\mathrm{x}, \mathrm{t})$

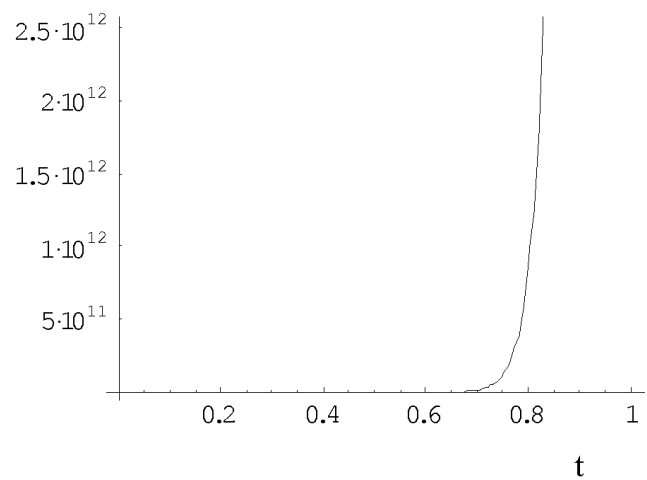

(c) $u(x, t)$

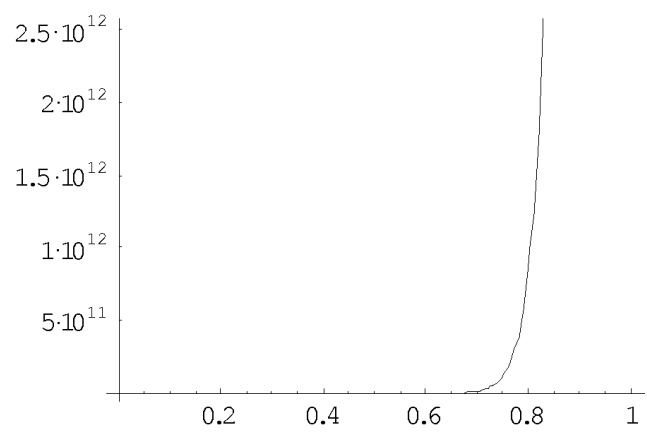

(b) $u(x, t)$

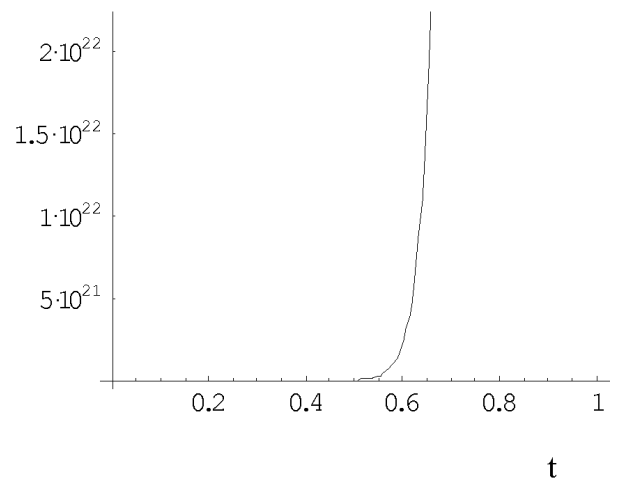

(d) $u(x, t)$

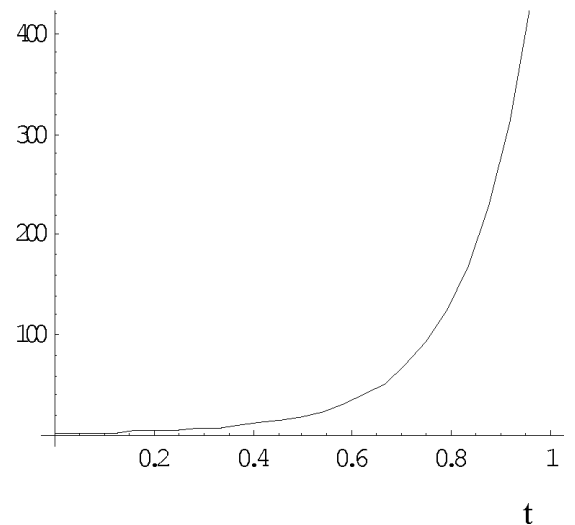

Fig. 1 Plot of $u(x, t)$ vs. $t$ at $x=1$ for $g(x)=\mathrm{e}^{x}$ and (a) $\alpha=\frac{1}{3}$, (b) $\alpha=\frac{1}{2}$, (c) $\alpha=\frac{2}{3}$, (d) $\alpha=1$.

Case IV: If $f(x)=-x$ and $g(x)=x^{6}$, then

$$
\begin{aligned}
& u(x, t)=x^{6}+\left(7 x^{6}+30 x^{4}\right) \frac{t^{\alpha}}{\Gamma(\alpha+1)} \\
& +\left(49 x^{6}+360 x^{4}+360 x^{2}\right) \frac{t^{2 \alpha}}{\Gamma(2 \alpha+1)} \\
& +\left(343 x^{6}+3270 x^{4}+5400 x^{2}+720\right) \frac{t^{3 \alpha}}{\Gamma(3 \alpha+1)} \\
& +\left(2401 x^{6}+26640 x^{4}+55440 x^{2}+11520\right) \frac{t^{4 \alpha}}{\Gamma(4 \alpha+1)} \\
& +\ldots=\sum_{r=0}^{\infty} \frac{k_{2}{ }^{r} t^{r \alpha}}{\Gamma(r \alpha+1)}=E_{\alpha}\left(k_{2} t^{\alpha}\right),
\end{aligned}
$$

where $k_{2}^{r}=\left[7^{r} x^{6}+30 \alpha_{r} x^{4}+360 \beta_{r} x^{2}+720 \delta_{r}\right]$, $\alpha_{r}=\frac{1}{2}\left[7 r-5^{r}\right], \beta_{r}=\frac{1}{70}\left[5.7^{r}-7.5^{r}\right]$, and $\delta_{r}=\frac{1}{150}$ $\cdot\left[3^{2} \cdot 5^{r}-5^{2} \cdot 3^{r}\right]+\frac{1}{1764}\left[3^{3} \cdot 7^{r}-7^{3} \cdot 3^{r}\right]$.

\section{Numerical Results and Discussion}

In this section, the numerical results of the displacement $u(x, t)$ for fractional Brownian motions with different values of $\alpha$, like $\alpha=\frac{1}{3}, \frac{1}{2}, \frac{2}{3}$, and for the standard diffusion equation with $\alpha=1$ have been evaluated by varying $t$ at $x=1$, when $f(x)=-x, g(x)=\mathrm{e}^{x}$ and $g(x)=\cos x$ while these results are depicted through Figures 1 and 2 .

It is shown in the results of Das [2], that the increase of $u(x, t)$ becomes higher as the integral power of $n$ of the initial condition $g(x)=x^{n}$ increases. In view of this result, an effort has been made to truncate the result series while calculating $u(x, t)$ for both $g(x)=\cos x$ and $g(x)=\mathrm{e}^{x}$. Since the presence of an exponential gives rise to an infinite series, for all practical considerations, we shall specify certain ranges for $t$ and $x$ as 
(a) $u(x, t)$

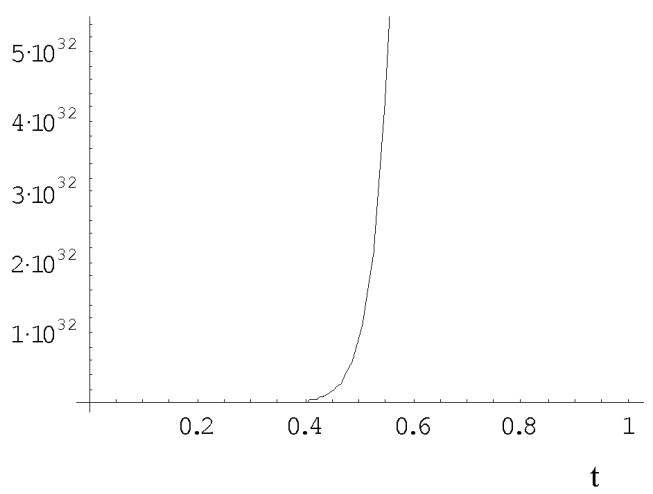

(c) $u(x, t)$

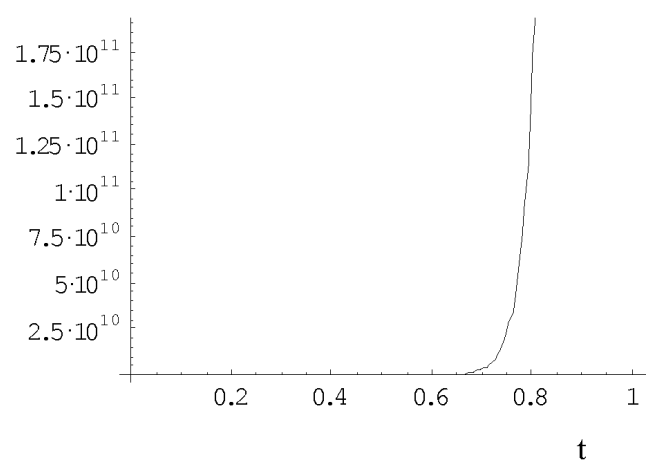

(b)

$$
u(x, t)
$$

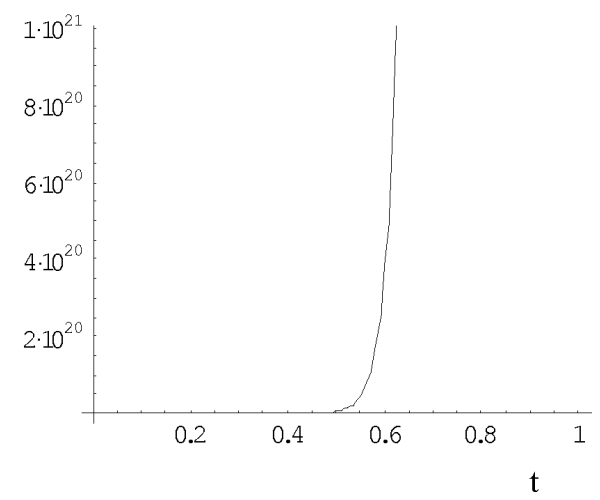

(d)

$$
u(x, t)
$$

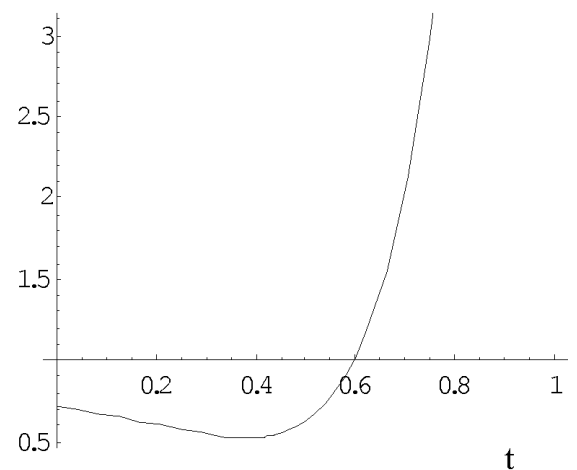

Fig. 2 Plot of $u(x, t)$ vs. $t$ at $x=1$ for $g(x)=\cos x$ and (a) $\alpha=\frac{1}{3}$, (b) $\alpha=\frac{1}{2}$, (c) $\alpha=\frac{2}{3}$, (d) $\alpha=1$.

in $(0<t<1)$ and $(0<x<1)$ during numerical computation. The results are shown graphically. It is seen from the figures that for both cases $u(x, t)$ increases with the increase in $t$ and decreases with the increase of $\alpha$, which is expected and conform with the decay of regular Brownian motion already determined by Giona and Roman [26].

\section{Conclusion}

There are two important goals that we have achieved by this study. First, employing the powerful HPM to investigate the general diffusion equation for different particular situations and, second, in showing its significant features.

HPM is a powerful mathematical tool which reduces the nonlinear problems to a set of ordinary differential equations to get the approximate analytical solution easily. In this study HPM requires less computational work to solve fractional diffusion equation than other reliable methods like VIM (Das [2]). Moreover it does not require small parameters in the equations which overcome the limitations of traditional perturbation techniques. This method is very convenient and effective in supplying quantitatively reliable results.

The most important outcome of the study is to present the scaling property of a fractional vibration equation to that of fractional Brownian motion (FBM) and the explanation of the decay of $u(x, t)$ with the increase of fractional time derivative $\alpha$ which has been shown both theoretically and numerically. The authors expect that the present study will considerably add value to the literature beyond the usual approach and this will considerably benefit the mathematicians and engineers working in this field. 


\section{Acknowledgements}

The authors express their sincere thanks to the referees for their valuable suggestions to improve of the paper.

[1] S. Saha Ray and R. K. Bera, Appl. Math. Comput. 174, 329 (2006).

[2] S. Das, Chaos, Solitons, and Fractals 42, 2074 (2009).

[3] J.H. He, Comp. Methods Appl. Mech. Eng. 167, 57 (1998).

[4] Z. M. Odibat and S. Momani, Int. J. Nonlinear Sci. Numer. Simul. 7, 27 (2006).

[5] S. Momani and Z. Odibat, Phys. Lett. A 365, 345 (2007).

[6] Z.Z. Ganji, D. D. Ganji, and H. Jafari, Topolog. Methods Nonlinear Anal. 31, 341 (2008).

[7] S. Das, Int. J. Nonlinear Sci. Numer. Simul. 9, 361 (2008).

[8] S. Momani, Z. Odibat, and I. Hashim, Topolog. Methods Nonlinear Anal. 31, 211 (2008).

[9] Z. Odibat and S. Momani, Topolog. Methods Nonlinear Anal. 31, 227 (2008).

[10] J. H. He, Comput. Methods Appl. Mech. Eng. 178, 257 (1999).

[11] J. H. He, Int. J. Nonlinear Mech. 35, 37 (2000).

[12] J. H. He, Phys. Lett. A. 347, 228 (2005).

[13] J. H. He, Chaos, Solitons, and Fractals 26, 695 (2005).

[14] J. H. He, Chaos, Solitons, and Fractals 26, 827 (2005).
[15] J. H. He, Int. J. Nonlinear Sci. Numer. Simul. 6, 207 (2005).

[16] J. H. He, Phys. Lett. A 350, 87 (2006).

[17] M. El-Shahed, Int. J. Nonlinear Sci. Numer. Simul. 6, 163 (2005).

[18] A. M. Siddiqui, R. Mahmood, and Q. K. Ghori, Int. J. Nonlinear Sci. Numer. Simul. 7, 7 (2006).

[19] A. M. Siddiqui, R. Mahmood, and Q. K. Ghori, Int. J. Nonlinear Sci. Numer. Simul. 7, 15 (2006).

[20] S. Momani and Z. Odibat, Comput. Math. Appl. 54, 910 (2007).

[21] J. H. He, Topolog. Methods Nonlinear Anal. 31, 205 (2008).

[22] J. H. He, Int. J. Modern Physics B 22, 3487 (2008).

[23] M. T. Darvishi and F. Khani, Z. Naturforsch. 63a, 19 (2008).

[24] M. M. Mousa and S. F. Ragab, Z. Naturforsch. 63a, 140 (2008).

[25] A. Belendez, M. L. Alvarez, D. I. Mendez, E. Fernandez, M. S. Yebra, and T. Belendez, Z. Naturforsch. 63a, 529 (2008).

[26] M. Giona and H. E. Roman, J. Phys. A: Math Gen. 25, 2093 (1992). 